\title{
The need for macrolides in hospitalised community-acquired pneumonia: propensity analysis
}

\author{
M. Paul*,\$, A.D. Nielsen\#,§, A. Gafter-Gvili*, E. Tacconelli", S. Andreassen", \\ N. Almanasreh ${ }^{+}$, E. Goldberg*, R. Cauda ", U. Frank ${ }^{+}$and L. Leibovici* on behalf of \\ the TREAT Study Group
}

ABSTRACT: The present study compared $\beta$-lactam macrolide ("combination") therapy versus $\beta$-lactam alone ("monotherapy") for hospitalised community-acquired pneumonia, using propensity scores to adjust for the differences between patients.

A prospective multinational observational study was carried out. Baseline patient and infection characteristics were used to develop a propensity score for combination therapy. Patients were matched by the propensity score (three decimal point precision) and compared with 30-day mortality and hospital stay. The propensity score was used as a covariate in a logistic model for mortality.

Patients treated with monotherapy $(n=169)$ were older (mean \pm SD age $70.6 \pm 17.3$ versus $65.0 \pm 19.6 \mathrm{yrs}$ ), had a higher chronic diseases score and a different clinical presentation compared with patients treated with combination therapy $(n=282)$. Unadjusted mortality was significantly higher with monotherapy (37 (22\%) out of 169 versus 21 (7\%) out of 282). Only 27 patients in the monotherapy group could be matched to 27 patients in the combination group using the propensity score. The mortality in these groups was identical, with three $(11 \%)$ demises each. The multivariable odds ratio for mortality associated with combination therapy, adjusted for the propensity score and the Pneumonia Severity Index, was 0.69 (95\% confidence interval $0.32-1.48$ ).

The benefit of combination therapy versus monotherapy cannot be reliably assessed in observational studies, since the propensity to prescribe these regimens differs markedly.

KEYWORDS: $\beta$-Lactams, combination, community-acquired pneumonia, macrolides, monotherapy, propensity score


uropean and North-American guidelines generally recommend a combination of a $\beta$-lactam drug in combination with a macrolide for patients admitted to hospital due to community-acquired pneumonia (CAP) [1-5]. There are two main reasons that underlie this recommendation. The first is to cover intracellular, "atypical" pathogens that do not respond to $\beta$-lactam drugs. Secondly, observational studies have shown that the outcome of patients with CAP [6-12] and bacteremic pneumococcal pneumonia [13-16] was better if treated with a $\beta$-lactam drug in combination with a macrolide compared with patients treated with a $\beta$-lactam drug alone. However, all these studies were nonrandomised. In vitro studies did not show synergy between $\beta$-lactams and macrolides $[17,18]$.

Patients treated for atypical pathogens are probably different $a$ priori from patients treated with a $\beta$-lactam drug alone. In their choice of treatment, physicians are likely to reflect common wisdom as to the presentation of atypical pathogens, i.e. younger patients, lower fever and leukocyte count, nonproductive cough, and certain patterns of infiltrate on the chest radiograph. Classical multi-variable techniques may not have been able to adequately adjust for the differences between the two patient groups, and the observed differences in outcomes may have been due to these a priori differences and not the higher efficacy of combination therapy.

Therefore, the present study addressed this question by analysing the outcomes of patients treated with a $\beta$-lactam in combination with a macrolide versus patients treated with a $\beta$-lactam alone, using propensity analysis.

\section{METHODS}

The present analysis included all CAP patients treated empirically with a combination of a
AFFILIATIONS

*Dept of Medicine E, Rabin Medical Center, Beilinson Campus and Sackler Faculty of Medicine, Tel-Aviv University, Tel-Aviv, Israel. \#University Center for Model-based Medical Decision Support, Aalborg University, Aalborg, Denmark.

"Dept of Infectious Diseases, Gemelli Hospital, Catholic University, Rome, Italy.

${ }^{+}$Dept of Clinical Microbiology and Hospital Hygiene, Freiburg University Hospital, Freiburg, Germany.

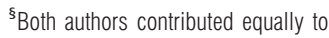
the manuscript.

CORRESPONDENCE

L. Leibovici, Dept of Medicine E Rabin Medical Center, Beilinson Hospital, 49100 Petah-Tiqva, Israel Fax: 97239376512

E-mail: leibovic@post.tau.ac.il

Received:

March 152007

Accepted after revision:

May 142007

\section{SUPPORT STATEMENT}

The TREAT project was funded by the European Union 5th framework, Information Society Technologies, contract No. IST-9999-11459. The funding source had no role in the study design, the collection, analysis and interpretation of data, in the writing of the report and in the decision to submit the manuscript for publication.

STATEMENT OF INTEREST None declared. 
$\beta$-lactam and a macrolide or with a $\beta$-lactam antibiotic alone, participating in the TREAT study $[19,20]$, a system for balancing antibiotic treatment against development of drug resistance. Patients were enrolled as part of a two-phase study (observational and interventional) designed to evaluate the effectiveness of TREAT, a computerised decision support system for antibiotic treatment of common bacterial infections among in-patients (Clinical-Trials.gov Identifier: NCT00233376). Patients were mainly admitted to medical wards and the study was conducted in three university-affiliated primary- and tertiary-care hospitals in Israel, Germany and Italy. During the observational phase, data were collected in Israel and Germany between June and December 2002, and in Italy between March and September 2003. The randomised controlled trial took place between May and November 2004 at all three sites. Research ethics committees in the three sites approved the study protocols.

\section{Inclusion and exclusion criteria}

The TREAT study included patients who: fulfilled the systemic inflammation response syndrome diagnostic criteria [21]; had a focus of infection; had shock compatible with septic shock; had febrile neutropenia; had been prescribed antibiotics (not for prophylaxis); and from whom blood cultures were drawn. The study excluded the following individuals: HIV-positive patients with a current (suspected or identified) opportunistic disease and/or AIDS-defining illness currently or within the previous 6 months; solid-organ or bone-marrow transplant recipients; patients $<18$ yrs of age; patients with suspected travel infections or tuberculosis; and pregnant females.

Patients fulfilling the inclusion criteria were prospectively identified by daily chart review. Within hours of admission data were collected on: demographics (e.g. age, sex, place of infection acquisition); background conditions (e.g. diabetes mellitus, chronic obstructive pulmonary disease, malignancy, chronic heart failure, chronic and acute renal failure, acute coronary syndrome, immunodeficiency); predisposing conditions (e.g. recent surgery) and devices (e.g. urinary catheter, intravenous catheter); presence of chills, temperature, pulse rate, systolic and diastolic blood pressure; focal signs and symptoms (e.g. cough, vomiting, rash); all available routine laboratory data (e.g. blood count, creatinine, urea, electrolytes, liver function tests); and chest radiography. At follow-up, 30 days after recruitment, data were collected on survival, final diagnosis, duration of hospital stay, fever days, duration of stay in the intensive care unit, treatment, adverse events and all microbiological results.

\section{Definitions and outcomes}

For the purpose of the present study CAP was defined as the presence of a new infiltrate on the admission chest radiograph of a patient fulfilling the TREAT inclusion criteria and presenting with symptoms/signs compatible with lower respiratory tract infection. The final main diagnosis at discharge or death of all patients included in the present cohort was pneumonia or related diagnoses. Empirical treatment was defined as the treatment administered in the first 2 days following hospital admission. Two main outcomes were assessed: 1) mortality, defined as all-cause mortality at 30 days following hospital admission; and 2) length of hospital stay.
Septic shock was defined as sepsis with hypotension despite adequate fluid resuscitation, along with the presence of perfusion abnormalities that may include, but are not limited to, lactic acidosis, oliguria, or an acute alteration in mental status. Functional capacity was measured on a scale of $0-3$ : $0=$ full functional capacity; $1=$ limited functional capacity; $2=$ limited in daily life activities; and $3=$ bedridden. The Charlson score was used to account for the presence of underlying chronic diseases [22]. The authors calculated the Pneumonia Severity Index (PSI) as predictor for mortality [23].

\section{Propensity analysis}

To perform a propensity analysis, the probability that a patient will be given combination therapy versus monotherapy was assessed using multivariate analysis. The model's predicted probability was used as the propensity score for each patient. The authors then matched patients receiving combination therapy versus monotherapy with similar propensity scores. This procedure provides two matched patient groups (combination versus monotherapy) that permit comparison of outcomes as in a randomised trial (pseudo-randomisation) [24]. The propensity score was used in two ways to correct for baseline disparities between groups. First, the authors compared outcomes between the matched patient groups (univariate). Secondly, the authors conducted a multivariate analysis for mortality among all patients adjusting for the propensity score within the model. For this analysis, patients outside the mutual range of the propensity scores for patients receiving combination therapy or monotherapy were excluded.

\section{Statistical analysis}

For univariate analysis, proportions were compared using a Fisher's exact test or Chi-squared test and continuous variables were compared using an unpaired t-test or Mann-Whitney Utest, as appropriate. Continuous variable values are reported as mean \pm SD. Univariate associations with a $p$-value $\leqslant 0.1$ were entered into the logistic regression analysis for the propensity score. Patients from the two groups were matched according to their propensity scores using a pre-defined precision of three decimal points. If more than one match was found, the patient to be included was selected at random. Length of stay in the two groups was compared by means of a General Linear Model (GLM), using the propensity score as a covariate. Model discrimination was assessed using the area under the receiver operating characteristics curve with $95 \%$ confidence intervals (CI).

\section{RESULTS}

In total, 611 patients with CAP were included in the TREAT study. The present study reports on 451 (74\%) patients who received a $\beta$-lactam drug alone $(n=169)$ or a $\beta$-lactam in combination with a macrolide $(n=282)$ as empirical treatment. Comparisons between the two groups as to the variables known at the time empirical treatment was decided upon are given in table 1 . The $\beta$-lactam drugs prescribed in the two groups are shown in table 2 . The pathogen causing pneumonia was documented in $28(17 \%)$ out of 169 patients receiving a $\beta$-lactam drug and in $32(11 \%)$ out of 282 patients receiving combination therapy $(\mathrm{p}=0.11)$. Legionella pneumonia was diagnosed in two patients receiving combination therapy. Blood cultures were positive in $10(6 \%)$ monotherapy versus 13 $(5 \%)$ combination therapy patients. Unadjusted 30-day mor- 
tality in the $\beta$-lactam group was $22 \%(n=37)$ versus $7 \%(n=21)$ in the $\beta$-lactam and macrolide group, univariate odds ratio (OR) for mortality with combination therapy was 0.29 (95\% CI $0.16-0.52 ; \mathrm{p}=0.0001)$. There was no difference in the mean $\pm \mathrm{SD}$ length of stay, $8.5 \pm 8.8$ versus $8.8 \pm 8.4$ days, respectively. Likewise, the mean length of stay was similar in the two groups when only patients alive on day 30 were included in the analysis.

In total, 14 variables were included in the logistic regression analysis to develop the propensity score (table 3). As expected, the propensity scores for the two groups differed markedly, $0.179 \pm 0.139$ versus $0.074 \pm 0.103$ for monotherapy versus combination therapy, respectively $(p<0.0001)$. For each of the three study locations the propensity score was significantly higher for patients receiving a $\beta$-lactam drug (data not shown). Only 27 patients in the $\beta$-lactam group could be matched to 27 patients in the $\beta$-lactam and macrolide group using the propensity score with a precision of three decimal points. The mortality in these groups was identical, with three $(11 \%)$ demises in each (OR (95\% CI) $1.0(0.2-5.5) ; p=1.0)$. The length of stay in hospital in the two groups was similar.

The PSI score predicted mortality well within the authors' cohort (area under curve $0.78 ; 95 \%$ CI $0.72-0.84 ; \mathrm{p}<0.001$ ). The treatment group was entered as a covariate to a logistic regression analysis for mortality with PSI. When patients outside the mutual range of the propensity scores for the two groups were excluded, 366 patients remained. Combination therapy remained significantly associated with lower mortality adjusted to PSI (OR 0.39, 95\% CI 0.19-0.79). However, when the propensity score (patients' predicted probability of being treated by combination therapy versus monotherapy) was entered to the model, the treatment arm no longer remained significantly associated with mortality (OR 0.69, 95\% CI 0.32 1.48). The PSI remained significantly associated with mortality in all models. Within this cohort, length of stay was not significantly different between groups (GLM model using the propensity score as a covariate).

The authors addressed the subgroup of the more severely ill patients in the cohort. Among all patients in PSI risk classes 4 or 5, all cause mortality was 34 (27\%) out of 128 versus 19 (11\%) out of 170 for monotherapy versus combination $(p=0.001)$, respectively. In the propensity-matched cohort the mortality for patients in the higher risk groups was three $(15 \%)$ out of 20 versus three $(16 \%)$ out of $19(p=0.95)$.

\section{DISCUSSION}

Patients who received a $\beta$-lactam alone for CAP were markedly different in the present study from those who received a combination of a $\beta$-lactam and a macrolide. The patients were older, chronic diseases were more common and a higher percentage of patients had chronic obstructive lung disease. Pneumonia presentation was different, with septic shock, disturbed consciousness and lobar or bronchopneumonic infiltrates more common among patients receiving $\beta$-lactam monotherapy. These differences were evident in the markedly different propensity scores. The gross mortality rate in this group was higher.

These differences impeded a propensity-matched analysis. When the authors tried to match patients from the two groups using the propensity score with a pre-defined precision of three decimal points, only 27 patients in each group (12\% of the cohort) could be matched. Among matched patients, mortality rates were identical. The difference in mortality between the two groups was nonsignificant when the propensity scores were used to adjust it in a logistic regression analysis. No differences were found in the length of stay.

Most observational studies have previously shown that the addition of a macrolide to $\beta$-lactams is associated with reduced

TABLE 1 Comparison between patients treated with a $\beta$-lactam alone versus patients treated with a $\beta$-lactam and a macrolide including variables known at the time empirical treatment was decided

\begin{tabular}{|c|c|c|c|}
\hline Variable & $\beta$-Lactam alone & $\beta$-Lactam and macrolide & p-value \\
\hline Subjects $n$ & 169 & 282 & \\
\hline Age yrs & $70.6 \pm 17.3$ & $65.0 \pm 19.6$ & 0.02 \\
\hline Nursing home residents & $16(9)$ & $10(4)$ & 0.01 \\
\hline Limited in daily life activities or bedridden & $65(60)$ & $43(40)$ & 0.0001 \\
\hline Charlson score & $1.5 \pm 0.9$ & $1.0 \pm 1.0$ & 0.0001 \\
\hline PSI score & $118.5 \pm 40.0$ & $98.5 \pm 40.9$ & $<0.001$ \\
\hline COPD & $44(26)$ & $54(19)$ & 0.1 \\
\hline Smoking & $30(18)$ & $71(25)$ & 0.09 \\
\hline Previous antibiotic treatment & $20(12)$ & $19(7)$ & 0.07 \\
\hline Duration of fever before admission days & $2.8 \pm 4.6$ & $2.1 \pm 2.5$ & 0.1 \\
\hline Chills & $15(9)$ & $54(19)$ & 0.003 \\
\hline Septic shock & $9(5)$ & $4(1)$ & 0.02 \\
\hline Acute disturbed consciousness & $36(21)$ & $20(7)$ & 0.0001 \\
\hline Pleuritic pain & $18(11)$ & $59(21)$ & 0.005 \\
\hline Cough & $64(38)$ & $184(65)$ & 0.0001 \\
\hline Infiltrate on chest radiograph: lobar or bronchopneumonia & $79(47)$ & 90 (32) & 0.001 \\
\hline
\end{tabular}

Data are presented as $n(\%)$ or mean \pm SD, unless otherwise stated. PSI: Pneumonia Severity Index; COPD: chronic obstructive pulmonary disease. 


\begin{tabular}{lcc} 
TABLE 2 & $\beta$-Lactam drugs prescribed in the two groups \\
\hline $\boldsymbol{\beta}$-Lactam drug prescribed & $\boldsymbol{\beta}$-Lactam alone & $\begin{array}{c}\boldsymbol{\beta} \text {-Lactam and } \\
\text { macrolide }\end{array}$ \\
\hline Subjects $\boldsymbol{n}$ & 169 & 282 \\
$\boldsymbol{\beta}$-Lactam and $\boldsymbol{\beta}$-lactamase inhibitor & $55(33)$ & $31(11)$ \\
Third generation cephalosporins & $71(42)$ & $151(54)$ \\
Second generation cephalosporins & $31(18)$ & $92(33)$ \\
Penicillins & $8(5)$ & $5(2)$ \\
Carbapenems & $4(2)$ & $3(1)$ \\
\hline
\end{tabular}

Data are presented as $n(\%)$, unless otherwise stated.

mortality among patients with CAP [6-16]. Few studies showed no effect [25-28]. Some features of these studies are described in table 4; most studies were retrospective. Significant differences were noted between patients receiving combination therapy versus monotherapy in most studies. However, outcome comparisons were adjusted most commonly to risk factors for mortality, not identical to the risk factors for the treatment regimen. Studies that showed similar characteristics for patients receiving monotherapy and combination therapy, or adjusting for the differences observed between the groups, showed no differences in outcomes [2628]. The authors believe that differences between study groups similar to those in the present cohort might have existed in former studies, and were not captured because the propensity for prescribing monotherapy versus combination therapy was not investigated. These differences are not necessarily captured when using risk factors for mortality to correct the association between treatment and mortality. When the two groups are divergent, with large areas that do not overlap, classical methods for multivariate adjusting might not be adequate [24].

A systematic review and meta-analysis of randomised controlled trials assessing the effect of empirical therapy covering atypical pathogens versus empirical regimens including only $\beta$-lactams has previously been carried out [30]. No difference was found in all-cause mortality overall (23 trials, 4,846 patients, relative risk $1.13,95 \%$ CI $0.82-1.54)$ or in trials including a macrolide in the atypical arm (five trials, 1,348 patients, relative risk $1.68,95 \%$ CI $0.86-3.29$, in favour of the $\beta$-lactam). However, a principal finding of this review [30] was that the addition of a macrolide or a quinolone to a $\beta$-lactam has never been assessed in a randomised controlled trial.

The present analysis was hampered by the small numbers of patients included. However, detailed data were prospectively and carefully collected using a uniform protocol in three hospitals in three countries (Germany, Italy and Israel). These data permitted a meticulous comparison between patients receiving monotherapy versus combination therapy. The differences between the patient groups were remarkable in the cohort. Differences might have been subtler in previous studies (table 4). The current study included patients admitted from nursing homes, excluded from some definitions of CAP. However, these patients consisted of $<7 \%$ of the cohort and were important to delineate the differences between patients receiving combination therapy versus monotherapy. The current authors did not assess fluoroquinolones, currently among the recommended regimens for hospitalised CAP [5], since only a few patients in the cohort received fluoroquinolones. Patients hospitalised in an intensive care unit, who may benefit preferentially from combination therapy, were also not included [11]. However, among the more severely ill patients in PSI risk classes 4 or 5 , the same trend was seen: higher

\section{TABLE 3 Logistic regression model for derivation of the propensity score}

\begin{tabular}{lccc} 
& Coefficient & p-value & OR (95\% Cl) \\
\hline Age $^{\#}$ & & & $0.996(0.981-1.011)$ \\
Nursing home residents & -0.004 & 0.579 & $0.20(0.04-1.00)$ \\
Limited in daily life activities or bedridden & -1.620 & 0.051 & $0.335(0.157-0.716)$ \\
Charlson score & -1.093 & 0.005 & $1.070(0.917-1.247)$ \\
Chronic obstructive lung disease & 0.067 & 0.392 & $0.407(0.215-0.772)$ \\
Smoking & -0.898 & 0.006 & $1.210(0.647-2.262)$ \\
Previous antibiotic treatment & 0.190 & 0.551 & $0.503(0.230-1.102)$ \\
Duration of fever before admission & 0.086 & $0.975(0.909-1.045)$ \\
Chills & -0.687 & 0.477 & $1.459(0.692-3.077)$ \\
Septic shock & -0.025 & 0.321 & $0.173(0.029-1.036)$ \\
Cough & 0.378 & 0.055 & $2.014(1.223-3.316)$ \\
Pleuritic pain & -1.756 & 0.006 & $1.652(0.798-3.423)$ \\
Acute disturbed consciousness & 0.700 & 0.177 & $0.630(0.286-1.388)$ \\
Infiltrate on chest radiograph: lobar or bronchopneumonia & 0.502 & 0.252 & $1.502(0.913-2.472)$ \\
Constant & -0.462 & 0.407 & 1.953 \\
\hline
\end{tabular}

OR: odds ratio; Cl: confidence interval. * : continuous variables, increment of $1 \mathrm{yr}$ for age; 1 point for Charlson score; 1 day for duration of febrile disease. Dependent variable: combination versus single $\beta$-lactam treatment. Hosmer and Lemeshow Chi-squared test $=11.0 ; 8$ degrees of freedom; $p=0.2$. Area under the receiver operator curve $0.77 ; 95 \% \mathrm{Cl} 0.72-0.82$ 


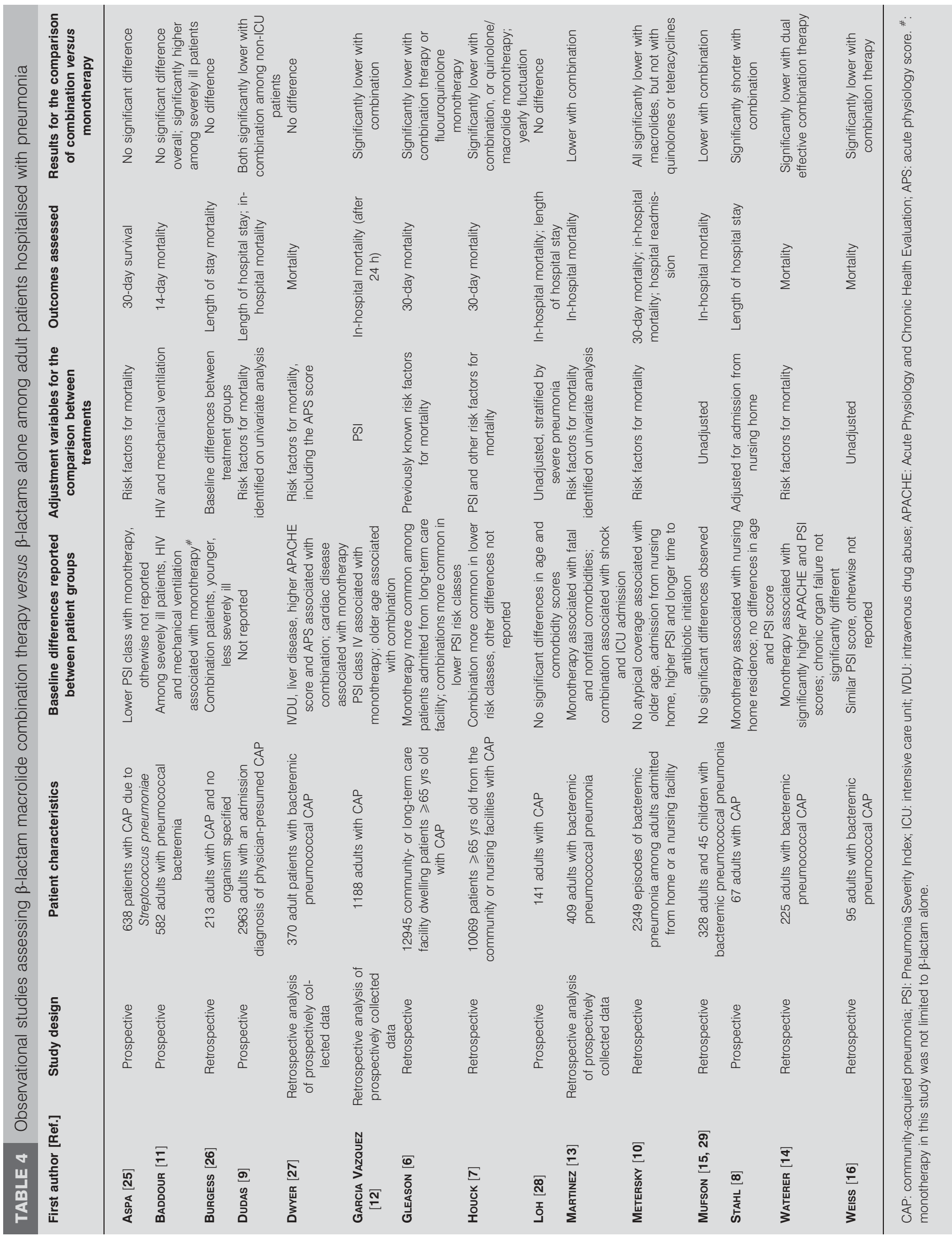


mortality among all patients with monotherapy compared with combination therapy, but no difference among the few patients remaining in the propensity-matched cohort.

It can be concluded that patients who receive a $\beta$-lactam alone for community-acquired pneumonia are markedly different from patients who receive a combination of a $\beta$-lactam and a macrolide. This difference precludes the use of observational studies to conclude on the advantage of one regimen over another. Excessive use of macrolides has consequences [31] and should be discouraged if the treatment does not improve the outcomes. A randomised controlled trial comparing a $\beta$-lactam drug with a combination of the same $\beta$-lactam and a macrolide for community-acquired pneumonia is urgently needed.

\section{ACKNOWLEDGEMENTS}

The following individuals contributed to the manuscript.

Conceived the project and basic concepts: L. Leibovici (Rabin Medical Center, Beilinson Hospital, Tel-Aviv, Israel) and S. Andreassen (University Center for Model-based Medical Decision Support, Aalborg University, Aalborg, Denmark).

Built the interface, database and supporting software: L. E. Kristensen, K. Falborg (both Judex A/S, Aalborg), A. Zalounina, A. D. Nielsen (both University Center for Modelbased Medical Decision Support).

Planned the clinical study: L. Leibovici, U. Frank (Freiburg University Hospital, Freiburg, Germany), E. Tacconelli (Gemelli Hospital, Catholic University, Rome, Italy), M. Paul (Rabin Medical Center), N. Almanasreh (Freiburg University Hospital), S. Andreassen, R. Cauda (Gemelli Hospital).

Collected data: A. Gafter-Gvili (Robin Medical Center), M. Paul, N. Almanasreh, E. Tacconelli, A. Cataldo (Gemelli Hospital), L. Vidal (Rabin Medical Center), M. Strehlein (Freiburg University Hospital), M. Cohen (Rabin Medical Center), E. Pokroy (Rabin Medical Center), R. Citton (Gemelli Hospital), D. Yahav, E. Skapa, S. Borok (all Rabin Medical Center)

Data analysis: M. Paul, A. D. Nielsen, L. Leibovici.

M. Paul and L. Leibovici had full access to all the data in the study and take responsibility for the integrity of the data and the accuracy of the data analysis.

\section{REFERENCES}

1 Mandell LA, Marrie TJ, Grossman RF, Chow AW, Hyland RH. Canadian guidelines for the initial management of community-acquired pneumonia: an evidencebased update by the Canadian Infectious Diseases Society and the Canadian Thoracic Society. The Canadian Community-Acquired Pneumonia Working Group. Clin Infect Dis 2000; 31: 383-421.

2 British Thoracic Society Standards of Care Committee. BTS Guidelines for the Management of Community Acquired Pneumonia in Adults. Thorax 2001; 56: Suppl. 4, IV1-IV64.

3 Hedlund J, Strålin K, Ortqvist A, Holmberg H, Community Acquired Pneumonia Working Group of the Swedish Society of Infectious Diseases. Swedish guidelines for the management of community-acquired pneumonia in immunocompetent adults. Scand J Infect Dis 2005; 37: 791-805.

4 Woodhead M, Blasi F, Ewig S, et al. Guidelines for the management of adult lower respiratory tract infections. Eur Respir J 2005; 26: 1138-1180.

5 Mandell LA, Wunderink RG, Anzueto A, et al. Infectious Diseases Society of America/American Thoracic Society consensus guidelines on the management of communityacquired pneumonia in adults. Clin Infect Dis 2007; 44: Suppl. 2, S27-S72.

6 Gleason PP, Meehan TP, Fine JM, Galusha DH, Fine MJ. Associations between initial antimicrobial therapy and medical outcomes for hospitalized elderly patients with pneumonia. Arch Intern Med 1999; 159: 2562-2572.

7 Houck PM, MacLehose RF, Niederman MS, Lowery JK. Empiric antibiotic therapy and mortality among Medicare pneumonia inpatients in 10 western states: 1993, 1995, and 1997. Chest 2001; 119: 1420-1426.

8 Stahl JE, Barza M, DesJardin J, Martin R, Eckman MH. Effect of macrolides as part of initial empiric therapy on length of stay in patients hospitalized with communityacquired pneumonia. Arch Intern Med 1999; 159: 2576-2580.

9 Dudas V, Hopefl A, Jacobs R, Guglielmo BJ. Antimicrobial selection for hospitalized patients with presumed community-acquired pneumonia: a survey of nonteaching US community hospitals. Ann Pharmacother 2000; 34: 446-452.

10 Metersky ML, Ma A, Houck PM, Bratzler DW. Antibiotics for bacteremic pneumonia: improved outcomes with macrolides but not fluoroquinolones. Chest 2007; 131: 466-473.

11 Baddour LM, Yu VL, Klugman KP, et al. Combination antibiotic therapy lowers mortality among severely ill patients with pneumococcal bacteremia. Am J Respir Crit Care Med 2004; 170: 440-444.

12 García Vázquez E, Mensa J, Martínez JA, et al. Lower mortality among patients with community-acquired pneumonia treated with a macrolide plus a $\beta$-lactam agent versus a $\beta$-lactam agent alone. Eur J Clin Microbiol Infect Dis 2005; 24: 190-195.

13 Martinez JA, Horcajada JP, Almela M, et al. Addition of a macrolide to a $\beta$-lactam-based empirical antibiotic regimen is associated with lower in-hospital mortality for patients with bacteremic pneumococcal pneumonia. Clin Infect Dis 2003; 36: 389-395.

14 Waterer GW, Somes GW, Wunderink RG. Monotherapy may be suboptimal for severe bacteremic pneumococcal pneumonia. Arch Intern Med 2001; 161: 1837-1842.

15 Mufson MA, Stanek RJ. Bacteremic pneumococcal pneumonia in one American City: a 20-year longitudinal study, 1978-1997. Am J Med 1999; 107: 34S-43S.

16 Weiss K, Low DE, Cortes L, et al. Clinical characteristics at initial presentation and impact of dual therapy on the outcome of bacteremic Streptococcus pneumoniae pneumonia in adults. Can Respir J 2004; 11: 589-593.

17 Johansen HK, Jensen TG, Dessau RB, Lundgren B, Frimodt-Moller N. Antagonism between penicillin and erythromycin against Streptococcus pneumoniae in vitro and in vivo. J Antimicrob Chemother 2000; 46: 973-980.

18 Lin E, Stanek RJ, Mufson MA. Lack of synergy of erythromycin combined with penicillin or cefotaxime 
against Streptococcus pneumoniae in vitro. Antimicrob Agents Chemother 2003; 47: 1151-1153.

19 Paul M, Andreassen S, Nielsen AD, et al. Prediction of bacteremia using TREAT, a computerized decision-support system. Clin Infect Dis 2006; 42: 1274-1282.

20 Paul M, Andreassen S, Tacconelli E, et al. Improving empirical antibiotic treatment using TREAT, a computerized decision support system: cluster randomized trial. J Antimicrob Chemother 2006; 58: 1238-1245.

21 Bone RC, Balk RA, Cerra FB, et al. Definitions for sepsis and organ failure and guidelines for the use of innovative therapies in sepsis. The ACCP/SCCM Consensus Conference Committee. American College of Chest Physicians/Society of Critical Care Medicine. Chest 1992; 101: 1644-1655.

22 Charlson ME, Pompei P, Ales KL, MacKenzie CR. A new method of classifying prognostic comorbidity in longitudinal studies: development and validation. J Chronic Dis 1987; 40: 373-383.

23 Fine MJ, Singer DE, Hanusa BH, Lave JR, Kapoor WN. Validation of a pneumonia prognostic index using the MedisGroups Comparative Hospital Database. Am J Med 1993; 94: 153-159.

24 Weitzen S, Lapane KL, Toledano AY, Hume AL, Mor V. Principles for modeling propensity scores in medical research: a systematic literature review. Pharmacoepidemiol Drug Saf 2004; 13: 841-853.
25 Aspa J, Rajas O, Rodriguez de Castro F, et al. Impact of initial antibiotic choice on mortality from pneumococcal pneumonia. Eur Respir J 2006; 27: 1010-1019.

26 Burgess DS, Lewis JS 2nd. Effect of macrolides as part of initial empiric therapy on medical outcomes for hospitalized patients with community-acquired pneumonia. Clin Ther 2000; 22: 872-878.

27 Dwyer R, Ortqvist A, Aufwerber E, et al. Addition of a macrolide to a ss-lactam in bacteremic pneumococcal pneumonia. Eur J Clin Microbiol Infect Dis 2006; 25: 518-521.

28 Loh LC, Quah SY, Khoo SK, Vijayasingham P, Thayaparan T. Addition of macrolide in treating adult hospitalized community-acquired pneumonia. Respirology 2005; 10: 371-377.

29 Mufson MA, Stanek RJ. Revisiting combination antibiotic therapy for community-acquired invasive Streptococcus pneumoniae pneumonia. Clin Infect Dis 2006; 42: 304-306.

30 Shefet D, Robenshtok E, Paul M, Leibovici L. Empirical atypical coverage for inpatients with community-acquired pneumonia: systematic review of randomized controlled trials. Arch Intern Med 2005; 165: 1992-2000.

31 Bergman M, Huikko S, Huovinen P, Paakkari P, Seppälä H, Finish Study Group for Antimicrobial Resistance (FiRe Network). Macrolide and azithromycin use are linked to increased macrolide resistance in Streptococcus pneumoniae. Antimicrob Agents Chemother 2006; 50: 3646-3650. 